\title{
El Parque Nacional del Manu, los pueblos indígenas y sus derechos: situación actual y tendencias
}

\section{Manu National Park, Indigenous People and their Rights: Current Situation and Trends}

\author{
Alfredo García Altamirano \\ https://orcid.org/0000-0003-1259-2042 \\ Federación Nativa del Río Madre de Dios y Afluentes \\ sahoemdd@gmail.com
}

Recibido: 05/02/2020 - Aceptado: 14/06/2020 - Publicado: 12/03/2021

\section{Resumen}

El presente artículo examina la situación actual de los derechos de los pueblos indígenas dentro de los parques nacionales en el país, a partir del análisis del caso del Parque Nacional del Manu (PNM). Se trata de poner al día este tema examinando su situación al interior de los parques nacionales, la categoría más elevada de protección de diversidad biológica del conjunto de categorías del Sistema Nacional de Áreas Naturales Protegidas del Perú (SINANPE).

\section{Palabras claves}

Áreas naturales protegidas; derechos indígenas; pueblos indígenas; parques nacionales; Parque Nacional del Manu.

\section{Abstract}

Based on the analysis of the Manu National Park (PNM) case, this article examines the current situation of the rights of indigenous peoples within national parks in the country. The aim is to update this issue by examining its situation within national parks, the highest category of protection of biological diversity of the National System of Protected Natural Areas of Peru (SINANPE).

\section{Keywords}

Protected areas; indigenous rights; indigenous people; national parks; Manu National Park.

Citar como: García Altamirano A. (2021). El Parque Nacional del Manu, los pueblos indígenas y sus derechos: situación actual y tendencias. Revista de antropología núm. 8: 37 - 60. http://dx.doi.org/10.15381/ antropologia.v0i8.19807 
El Parque Nacional del Manu, establecido en 1973, es el área natural protegida más emblemática del país, debido a los impresionantes records de diversidad biológica allí documentados. De acuerdo con su Plan Maestro 2013-20181, contiene el $10 \%$ de las especies de aves del mundo, el 5\% de los mamíferos, y una vasta cantidad de peces, reptiles, anfibios e invertebrados. Además, contiene al menos 50 especies animales globalmente amenazadas, de acuerdo con la lista roja de la International Union for Conservation of Nature (IUCN); se encuentra dentro de un hotspot de biodiversidad, el de los Andes Tropicales, la región más rica y diversa del mundo, designado por Conservación International (CI); y allí están dos de las 200 ecorregiones con alta prioridad mundial para la conservación: las yungas peruanas y los bosques húmedos de la Amazonía suroccidental. Esto le ha valido obtener reconocimientos como el ser declarado en 1987 Patrimonio Natural de la Humanidad, y recibir en 1997 el estatus de Reserva de Biosfera, el cual fue otorgado por UNESCO (SERNANP, 2014).

Sin embargo, es poco conocido que el PNM se estableció en territorios indígenas y que está ocupado y habitado por varios pueblos indígenas amazónicos, incluyendo pueblos indígenas aislados. $\mathrm{Y}$, menos conocido aun, es el tratamiento que se observa o que se da a los derechos indígenas dentro de las áreas naturales protegidas (ANP). Un informe de la Defensoría del Pueblo se dedicó a este tema, aunque no circunscrito al PNM sino a 16 ANP existentes en la Amazonía peruana, todas creadas en territorios de pueblos indígenas (Camino, 2000). Con atención al caso del PNM, otros trabajos posteriores han examinado las relaciones y tensiones existentes entre el área protegida y los pueblos indígenas que lo habitan. Entre ellas están Shepard et al. (2010, 2013), Rodríguez (2013, 2018), Rodríguez y Feather (2014) y Ráez (2018).

El tema de la relación entre parques y pueblos no es nuevo en las ciencias sociales. En general, para observadores y científicos que provienen del campo tanto de las ciencias naturales como de las ciencias sociales, existen diferentes lecturas de esta realidad. Grosso modo, se puede decir que hay dos orillas desde las cuales se hacen estas observaciones: quienes miran la realidad indígena desde la óptica de la protección de la diversidad biológica, estrictamente apegados a las prescripciones de las escuelas de conservación proteccionistas, y quienes se esfuerzan por alcanzar una lectura más holística de la condición indígena dentro de áreas protegidas, procurando un enfoque más integral de la relación parquespueblos. Además, en ambas orillas hay matices de estas observaciones.

El examen de los pueblos indígenas que viven dentro del PNM y sus derechos cubre una amplia gama de asuntos. He seleccionado lo que considero son los tópicos más relevantes de su situación actual: (1) los pueblos indígenas del PNM; (2) la crisis de la relación parques-pueblos; y (3) planes antropológicos y provisiones de derechos indígenas. Como tendencias examino: (4) la autogestión, los derechos

${ }^{1}$ Existe una versión de este Plan para el periodo 2019-2023 que aún está pendiente de publicación. 
y los puntos de vista indígenas; y (5) los escenarios futuros y la gobernanza del PNM. Basado en ello, trazamos algunas conclusiones sobre la pregunta inicial.

La preparación de este articulo ocurrió en el marco de un proyecto de investigación impulsado por la Federación Nativa del Río Madre de Dios y Afluentes (FENAMAD) y el Centro de Investigaciones para América Latina y El Caribe (CERLAC) de la Universidad de York (Canadá). El proyecto se enfocó en el examen de la situación de la territorialidad ese'eja pero incluyó un estudio complementario para el caso de los matsigenka del PNM. Las observaciones de campo que alimentaron este artículo se llevaron a cabo en el contexto de misiones de campo de FENAMAD a las comunidades matsigenka del PNM, ocurridos en octubre de 2016 y entre febrero y marzo de 2017. El autor formó parte del equipo técnico que FENAMAD movilizó para realizar estudios socioeconómicos que buscaron sustentar el reconocimiento como comunidades nativas a los asentamientos matsigenka Maizal y Cacaotal. Otras observaciones posteriores han ocurrido como parte del trabajo del autor como parte del equipo técnico de FENAMAD desde enero del 2018.

\section{LOS PUEBLOS INDÍGENAS DEL PNM}

El PNM se estableció sobre una superficie de 1'532,806 ha, en una combinación de ecosistemas que comprende el páramo andino y la selva baja. El establecimiento del PNM implicó que se cierren las operaciones de extracción de madera en toda la cuenca del río Manu². En la Zona Reservada Manu se establecieron las operaciones de turismo del PNM. Su condición provisional para determinar su uso futuro se extendió hasta el año 2002 cuando se dispuso, mediante el Decreto Supremo $N^{\circ}$ 045-2002-AG, su recategorización, en la figura de ampliación de la extensión del PNM a 1’716,295.22 ha (SERNAMP, 2014).

En 1968, antes de crearse el PNM, existía en Tayakome una misión evangélica del Instituto Lingüístico de Verano (ILV). Durante el proceso de constitución del PNM (1970-1973), André Marcel d'Ans, lingüista y antropólogo belga, desarrolló investigaciones sobre los indígenas del Manu en el marco de un convenio entre el Ministerio de Educación y la Universidad Nacional Mayor de San Marcos (Helberg, 2001). Entonces, los indígenas matsigenka que vivían en el río Manu ya estaban articulados a la economía de mercado, aunque en condiciones desventajosas, pues lo hacían principalmente como mano de obra para los patrones madereros, y también como proveedores de leña, pieles, carne de monte, entre otros, que iban a manos de dichos patrones madereros y otros comerciantes establecidos entonces en Boca Panagua. El corte del circuito

\footnotetext{
${ }^{2}$ En 1973, había tres empresas madereras extrayendo madera legalmente (Vracko, Reyner y Otsuka), además de varios extractores informales de pequeña escala. Se les otorgó dos ańos de plazo para retirarse, además del derecho a obtener contratos forestales en otros lugares. Se retiraron en 1975 (Thomas Moore, comunicación personal, 2016; Héctor Sueyo, comunicación personal, 2016).
} 
económico mercantil con el exterior fue un hecho impuesto desde afuera, al momento de establecerse el PNM (Helberg, 2001).

Además de los matsigenka, en la cuenca baja del río Manu vivían los yine, articulados a las actividades de extracción de madera y pequeños fundos agropecuarios que proveían aguardiente y otros insumos a los madereros. Ambos pueblos indígenas tienen diversos registros de su presencia en la cuenca del río Manu, mucho antes del establecimiento del ANP. Mientras los matsigenka se han movido entre el PNM y otras localidades matsigenka en la cuenca del Urubamba, donde tienen parientes (y viceversa, del Urubamba al Manu), los yine del río Manu que habitaban hasta hace 50 ańos el tramo inferior del río Manu, desde el río Panahua, aguas abajo, ahora están establecidos en las comunidades nativas de Diamante, ubicada en la margen izquierda del río Alto Madre de Dios, a la altura de la desembocadura del río Manu, y de Isla de los Valles, detrás del actual centro poblado mestizo de Boca Manu.

El primer Plan Maestro del PNM fue elaborado en 1985 y contenía una sección llamada Antropología que reunía los resultados de las investigaciones realizadas por d'Ans. El registro de pueblos indígenas en la parte baja del PNM se ha afinado desde este primer Plan Maestro, especialmente con los sucesivos Planes Antropológicos del $\mathrm{PNM}^{3}$. De acuerdo con estos, se distinguen al interior del PNM, dentro de la familia lingüística arawak, a los pueblos matsigenka, matisgenka-nanti y yine. En la familia lingüística pano sólo se reconoce a un grupo étnico, los yora. En la zona de selva inmediatamente adyacente al PNM, se distinguen a los yine, igualmente de la familia lingüística arawak, y varios subgrupos harakbut de la familia lingüística harakbut.

En la Figura 1 se presenta la distribución de localidades indígenas en la parte amazónica $^{4}$ del PNM y la Reserva de Biósfera Manu (RBM), y en la Tabla 1 se detalla la información de los asentamientos y localidades indígenas amazónicas contenidas en la Figura 1.

Sobre la base de los registros antropológicos antes citados, distingo tres condiciones para los pueblos indígenas que habitan el PNM. Una de ellas, relativo a pueblos indígenas aislados, señala su condición de autónomos, no articulados o integrados a la sociedad nacional. Actualmente, el pueblo aislado más conocido es de habla arawak, localmente denominado mashko piro ${ }^{5}$, avistados desde los ańos ochenta entre los ríos Pinkén y Alto Madre de Dios y también entre los ríos Manu y Los Amigos. Un grupo de tres mujeres mashko piro vivieron muchos años frente al puesto de control y vigilancia de Pakitza,

${ }^{3}$ Especialmente las versiones de 1997, 2002 y 2007.

${ }^{4}$ Tambien existen algunos asentamientos de poblacion andina en la zona de Callanga (antiguas haciendas) que quedaron dentro del PNM cuando este se creó.

${ }^{5}$ El PNM es una parte del corredor territorial del pueblo indígena aislado mashco piro que comprende la Reserva Territorial Madre de Dios, la Concesion de Conservación Los Amigos, el Parque Nacional Alto Purús y otras áreas al otro lado de la frontera con Brasil (Huertas, 2015). 
hasta que, vía relaciones matrimoniales con matsigenka y yine, se establecieron en las comunidades nativas de Shipetiari y Diamante (Huertas, 2002), entre el 2000 y el 2001, aproximadamente. Desde el 2011, los mashko piro son avistados con más frecuencia en la margen izquierda del río Alto Madre de Dios y otras áreas como la cuenca del rio Las Piedras.

Figura 1.

Asentamientos indígenas en el PNM y la Reserva Biósfera Manu.

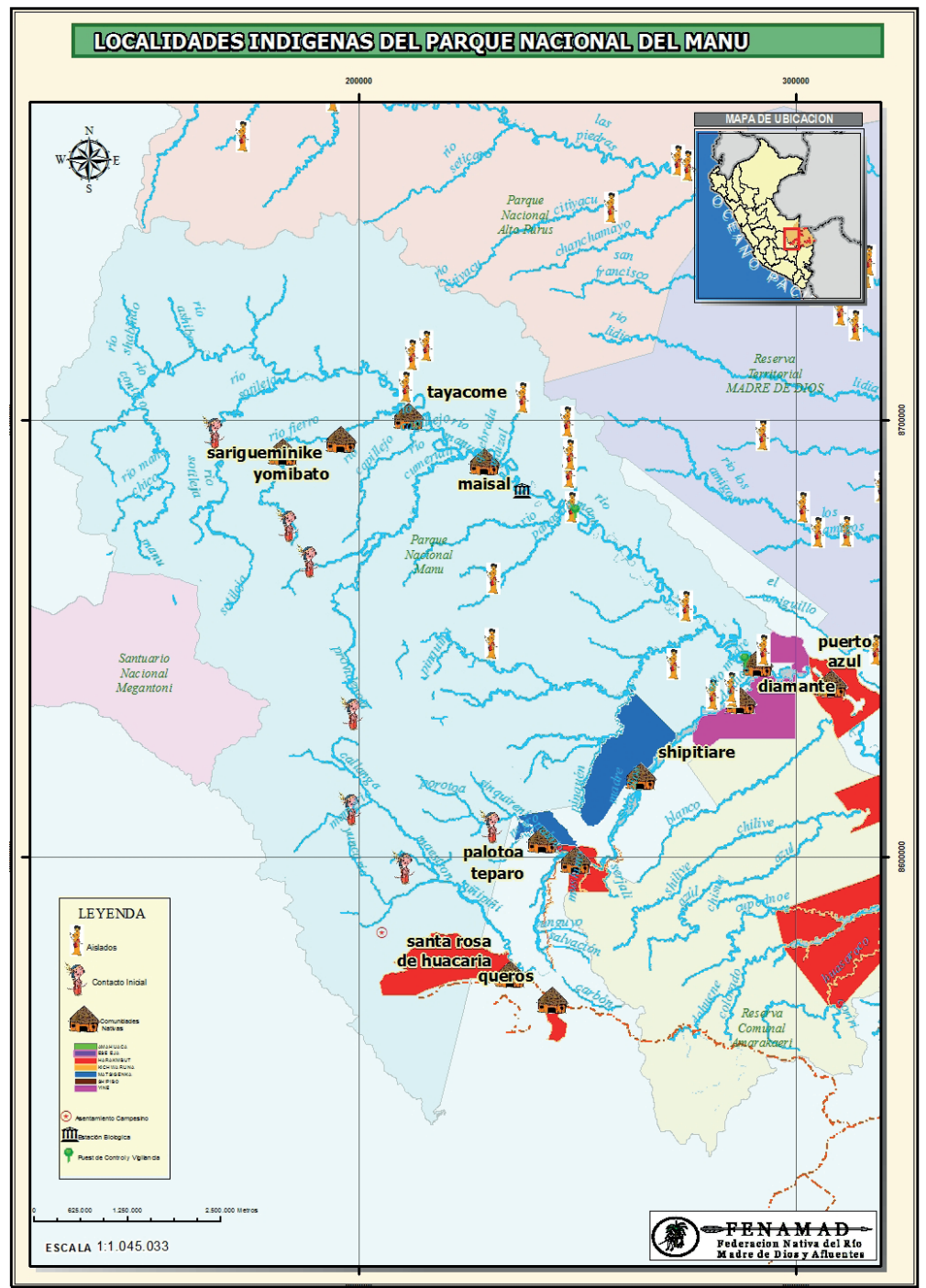

Fuentes: CTAR-IIAP (1999), FENAMAD (2013), García (1998) y Shepard et al. (2010)

${ }^{6}$ Los planes antropológicos del PNM de 1997, 2002 y 2007 han conservado registros de otros pueblos indígenas no identificados. Los matsigenka de Yomibato hablan de amahuaca aislados, aunque no se cuenta con mayores precisiones sobre ellos. 
El Parque Nacional del Manu, los pueblos indígenas y sus derechos: situación actual y tendencias

Tabla 1.

Asentamientos indígenas en el PNM y Reserva Biósfera Manu.

\begin{tabular}{|c|c|c|c|c|}
\hline Situación & Río(s) & Localidad & $\begin{array}{l}\text { Familia } \\
\text { Lingüística }\end{array}$ & Grupo Étnico \\
\hline Ais & \multicolumn{2}{|c|}{$\begin{array}{l}\text { Pinkén, Pinquencillo, Panahua, Manu (margen izquierda, } \\
\text { entre Boca Manu y Panagua) }\end{array}$} & Arawak & “Mashco Piro" \\
\hline Ais & \multicolumn{2}{|c|}{ Alto Madre de Dios (frente a Diamante) } & Arawak & "Mashco Piro" \\
\hline $\mathrm{Ci}$ & \multicolumn{2}{|c|}{ Manu (cabeceras, margen izquierda) } & Pano & Yora \\
\hline $\mathrm{Ci}$ & \multicolumn{2}{|c|}{ Alto Sotileja, Alto Yomibato, Alto Cumerjali } & Arawak & Matsigenka \\
\hline $\mathrm{Ci}$ & Palotoa & Abaroa & Arawak & Matsigenka \\
\hline $\mathrm{Ci}$ & \multicolumn{2}{|c|}{ Piñipiñi, Nystrom y Amalia } & Arawak & Matsigenka \\
\hline $\mathrm{Cn}$ & Manu & Tsirerishi (Maizal) & Arawak & Matsigenka \\
\hline $\mathrm{Cn}$ & Manu & Tayakome & Arawak & Matsigenka \\
\hline $\mathrm{Cn}$ & Manu & Sarigueminiki (Cacaotal) & Arawak & Matsigenka \\
\hline Cn & Yomibato, Manu & Yomibato & Arawak & Matsigenka \\
\hline \multirow{2}{*}{$\mathrm{CN}$} & \multirow{2}{*}{ Piñipiñi } & \multirow{2}{*}{ Santa Rosa de Huacaria } & Arawak & Matsigenka \\
\hline & & & Harakbut & Wachiperi \\
\hline $\mathrm{CN}$ & Palotoa & Palotoa-Teparo & Arawak & Matsigenka \\
\hline $\mathrm{CN}$ & Alto Madre de Dios & Shipetiari & Arawak & Matsigenka \\
\hline $\mathrm{CN}$ & Alto Madre de Dios & \multirow{2}{*}{ Shintuya } & Harakbut & Arakbut \\
\hline $\mathrm{CN}$ & Shintuya & & Harakbut & Wachiperi \\
\hline $\mathrm{CN}$ & Alto Madre de Dios & Diamante & Arawak & Yiné / Matsigenka \\
\hline $\mathrm{CN}$ & Manu & Isla de Los Valles & Arawak & Yiné \\
\hline
\end{tabular}

Notas: acotado a los periodos de observación según fuentes consultadas

Abreviaturas:
Ais Pueblos indígenas aislados
$\mathrm{Ci}$ Pueblos indígenas en contacto inicial
$\mathrm{Cn}$ Comunidad nativa reconocida
$\mathrm{CN} \quad$ Comunidad nativa reconocida y con tierras demarcadas y tituladas

Datos de pueblos indígenas en aislamiento y contacto inicial provenientes de: CTAR-IIAP (1999), Dávila y Montoya (1999), FENAMAD (2002), Huertas (2002), Rummenhöller et al. (1997a, 1997b), Shepard (1996a, 1996b), Shepard et al. (2010)

Datos de comunidades nativas: MINAGRI (2000)

Cuadro de elaboración propia, 2020.

Una segunda condición se refiere a los pueblos indígenas en contacto inicial. Este contacto ocurre mayormente con otros paisanos suyos, en comunidades que tienen cierta articulación con la sociedad nacional. Son los casos de asentamientos matsigenka tradicionales y dispersos que se ubican, por ejemplo, en las cabeceras de los ríos Sotileja y Cumerjali, y que se comunican con sus paisanos y parientes de la comunidad nativa de Yomibato. Una situación similar ocurre con matsigenka de los ríos Piñi Piñi y Palotoa, afluentes del río Alto 
Madre de Dios, que se comunican con miembros de la comunidad wachiperimatsigenka de Santa Rosa de Huacaria y la comunidad matsigenka de Palotoa, respectivamente. Antes llamados kugapakori, prefieren como autodenominación llamarse matsigen $\mathrm{ka}^{7}$.En condición de pueblo indígena en contacto inicial se encuentra una fracción del pueblo yora, de habla pano, cuya presencia se registró entre los ańos setenta y ochenta al norte del PNM. Conocidos como nahua en el Ucayali y también en el PNM, fueron violentamente "contactados" en los ochenta desde el Ucayali (Reynoso y Helberg, 1986; Wahl, 1988). Parte de los sobrevivientes permanecieron en las cabeceras del río Manu hasta que migraron a Santa Rosa de Serjali, en la cuenca del Urubamba, fuera del PNM. En las cabeceras del rio Manu conservan chacras (Huertas, 2015).

Una tercera condición se refiere a los asentamientos locales indígenas articulados a la sociedad nacional, como es el caso de las comunidades nativas matsigenka Tayakome y Yomibato, dentro del PNM. El patrón de asentamiento tradicional matsigenka es disperso. Su conformación, como comunidades, implicó un nucleamiento en aldeas relativamente concentradas. Sin embargo, dentro de ese nucleamiento reproducen, en pequeña escala, la dispersión tradicional. Fueron reconocidos legalmente como comunidades nativas en 1988. En el 2019, los asentamientos matsigenka Tsirerishi (antes conocido como Maizal) y Sarigeminiki (antes conocido como Cacaotal), obtuvieron su reconocimiento como comunidades nativas. En 1985 se tituló la comunidad nativa de Santa Rosa de Huacaria, ubicada entre los ríos Piñi Piñi y Tono. La escasa información precisa sobre los límites del PNM de entonces llevó a que alrededor del 60\% del área titulada se encuentre dentro del PNM (Rummenhöller et al., 1997b).

Es pertinente advertir que Ráez caracteriza a las comunidades matsigenka del PNM (Tayakome, Yomibato, Tsirerishi, Sarigueminiki) como de "contacto inicial" (2018, p. 19), colocándolas al mismo nivel que otras comunidades que si observan esta cualidad como los matsigenka-nanti de Montetoni o los matsigenka de Cumerjali y Sotileja. Es cierto que, respecto del entorno occidental, el manejo de los códigos occidentales por parte de dichas comunidades matsigenka es escaso. Sin embargo, en mi perspectiva, es más influyente la articulación con la economía de mercado, la escuela, los servicios de salud y otros a los que acceden las comunidades matsigenka, todos elementos ausentes entre los matsigenka más remotos.

\section{LA CRISIS DE LA RELACIÓN PARQUE-PUEBLOS}

Desde su creación, el PNM ha enfrentado varias crisis, en particular con respecto a la relación entre el parque y sus poblaciones indígenas. Pero, a la vez, han sucedido, y suceden, varias otras crisis y tensiones con diversos actores sociales e institucionales, internos y externos, que se han yuxtapuesto e influido en el

\footnotetext{
${ }^{7}$ Dos asentamientos matsigenka-nanti, Montetoni y Marankiato, se ubican al oeste del PNM, en la actual Reserva Territorial Kugapakori, Nahua Nanti y otros.
} 
curso de las propias crisis y tensiones entre el parque y sus poblaciones indígenas. Por ejemplo, en 1968 existía una misión evangélica del ILV en Tayakome, la cual contaba con un aeródromo a su cargo. Con el proceso de creación del PNM, el ILV mudó dicha misión fuera del Manu, lo que influyó en el cierre de su servicio sanitario, causando grandes estragos entre la población matsigenka de Tayakome. Al mismo tiempo, el primer puesto de control y vigilancia (PCV) se estableció en las inmediaciones de la antigua misión del ILV en Tayakome. Los abusos y violaciones a mujeres y otras trapacerías cometidas por los guardaparques -que entonces tenían formación militar y andaban armados- contra los indígenas matsigenka, llevó a protestas y una acción de autodefensa indígena que expulsó este PCV a otro sitio denominado Pakitza, aguas abajo (Shepard et al., 2010).

Una mirada comprensiva de los distintos momentos críticos en las tensiones entre indígenas y Estado (en la figura del PNM), las reúno en la Tabla 2. En los siguientes acápites habrá lugar de comentar, con más detalle, algunas de estas crisis. Por el momento, la intención aquí es señalar los conflictos registrados y documentados, conforme a las fuentes consultadas.Quien más se ha esforzado por entender los conceptos que sustentan las políticas de gestión del PNM es Rodríguez $(2013,2018)$. En ultima instancia, en la base de los proyectos estatales de áreas protegidas "está como principal sostén epistemológico la división naturaleza/sociedad", donde se piensa a esta última como "fuera de la naturaleza", en base a lo cual se "sentó las bases, a su vez, para el desarrollo de la idea de una humanidad destructiva categorizada como amenaza, analíticamente externa al mundo natural” (Rodríguez, 2013, pp. 7-8, énfasis original).

Esta idea de que cualquier población humana, incluyendo los pueblos indígenas, son una amenaza para garantizar la reproducción de procesos biológicos esenciales, parece ser una premisa que se ha seguido en el establecimiento de ANP alrededor del mundo. El concepto de parque nacional, según Harroy (1972):

surgió en los Estados Unidos de América durante la expansión del lejano oeste. En 1872 el congreso de los Estados Unidos utilizó por primera vez la denominación parque nacional para reservar y separar de cualquier impacto de colonización y ocupación humana un área de 899,139 ha, en el curso superior del río Yellowstone, Wyoming, 'dedicado y establecido como un parque público o lugar de esparcimiento para el beneficio y placer de la gente' (Citado por Rummenhöller, 1995, p. 65).

Señala este autor que "los fundadores utilizaron la denominación parque para referirse a un 'área de beneficio de visitantes' y 'acceso público para disfrutar la naturaleza'. El término nacional significaba que se trataba de un asunto de interés nacional por lo cual sólo el gobierno federal debía manejarlo" (Rummenhöller, 1995, p. 65, énfasis original). Pero Yellowstone no era un bosque "vacío" sino territorio indígena de los shoshone, crow y blackfeet (Rummenhöller, 1995). Cien años después de Yellowstone, había 1,200 parques nacionales en todo el 
mundo (Gray, 1992). De allí la intensa discusión por los impactos de la protección de la diversidad biológica con el establecimiento de parques expulsando a sus habitantes originarios de ellos, situación más que contraproducente (Gray, 1992).

Tabla 2.

Crisis en la relación PNM y pueblos indígenas.

\begin{tabular}{|c|c|c|}
\hline Año & $\begin{array}{l}\text { Tipificación de la } \\
\text { crisis }\end{array}$ & Principales elementos \\
\hline \multirow{3}{*}{$1973-1985$} & \multirow{3}{*}{ La crisis creciente } & $\begin{array}{l}\text { Vacío de sistema sanitario y médico en Tayakome por salida del ILV e } \\
\text { incremento de mortalidad infantil. }\end{array}$ \\
\hline & & $\begin{array}{l}\text { Las nuevas escuelas establecidas por la misión dominica en Tayako- } \\
\text { me y Yomibato impulsan mayor sedentarización, aculturación y } \\
\text { asimilación a la sociedad nacional. }\end{array}$ \\
\hline & & $\begin{array}{l}\text { Indígenas matsigenka explotados fuera del PNM y nulos beneficios } \\
\text { del turismo para las comunidades }\end{array}$ \\
\hline \multirow{3}{*}{$1985-1997$} & \multirow{3}{*}{$\begin{array}{l}\text { Reacción ante la } \\
\text { crisis }\end{array}$} & $\begin{array}{l}\text { La administración del PNM cuenta por única vez con un antropólogo } \\
(1985-87) \text { y formula política antropológica }\end{array}$ \\
\hline & & $\begin{array}{l}\text { Propuesta de proyecto Albergue Matsigenka formulada por el Centro } \\
\text { para el Desarrollo del Indígena Amazónico (CEDIA), rechazada por } \\
\text { INRENA. }\end{array}$ \\
\hline & & $\begin{array}{l}\text { Amplio diagnóstico participativo de la realidad social dentro y fuera } \\
\text { del PNM }\end{array}$ \\
\hline \multirow{3}{*}{1997 - 2010} & \multirow{3}{*}{$\begin{array}{l}\text { Acercamiento, } \\
\text { negociación y } \\
\text { Albergue Matsi- } \\
\text { genka }\end{array}$} & $\begin{array}{l}\text { Proyecto Albergue Matsigenka cooptado por INRENA; construcción } \\
\text { (1997-1998) con apoyo de APECO y con recursos de FANPE-GTZ. }\end{array}$ \\
\hline & & $\begin{array}{l}\text { Relación positiva entre el PNM y las comunidades matsigenka (1998- } \\
\text { 2000) }\end{array}$ \\
\hline & & $\begin{array}{l}\text { Otros operadores turísticos logran permiso para construir sus } \\
\text { propios albergues; el Albergue Matsigenka se debilita }\end{array}$ \\
\hline \multirow{6}{*}{$2011-2019$} & \multirow{6}{*}{$\begin{array}{l}\text { Nueva crisis en } \\
\text { curso }\end{array}$} & Mashco piro: presencia permanente en Alto Madre de Dios \\
\hline & & Fracaso financiero del Albergue Matsigenka (2013) \\
\hline & & $\begin{array}{l}\text { Nuevo convenio con SZF: Persistencia en políticas de vaciar el parque } \\
\text { de personas }\end{array}$ \\
\hline & & $\begin{array}{l}\text { Aumento del compromiso político y comercial de la población indí- } \\
\text { gena con el mundo de afuera: presión para la extracción comercial }\end{array}$ \\
\hline & & $\begin{array}{l}\text { Plan Maestro 2013-2018: plan antropológico como actividad opcio- } \\
\text { nal }\end{array}$ \\
\hline & & Gobernador Otzuka impulsa carretera Nuevo Eden-Boca Manu[1] \\
\hline
\end{tabular}

[1] Esta carretera, que en parte funciona y en parte está paralizada, fue lanzada como una iniciativa expresamente dirigida en contra del PNM por su promotor e indirectamente contra los pueblos indígena en situación de aislamiento y contacto inicial (PIACI). Existen grupos de interés promoviendo actualmente que este proyecto continúe.

Fuentes: Shepard et al. (2010, 2013); Rodríguez (2013, 2018). Elaboración propia, 2020.

Por consiguiente, encarrilado en este patrón mundial que siguió la creación de parques nacionales, no es nada extrańo que el primer plan maestro del PNM de 1986 haya propugnado la reubicación de poblaciones indígenas fuera del mismo, aunque sujetas a la restricción de hacerlo cuando estas se modernizaran: 
se pretende que los diferentes grupos nativos se mantengan dentro del Parque cuando lleven una vida de acuerdo a su cultura tradicional; aquellos que opten por un modo 'civilizado', es decir de aculturación avanzada, tendrán que salir de los límites de la Unidad (Ríos et al., 1986, p. 87).

La aculturación y asimilación de valores occidentales por parte de los pueblos indígenas deviene en factor y causal para reubicar poblaciones indígenas fuera del PNM. John Terborgh, reputado ornitólogo que trabajaba en la Estación Biológica de Cocha Cashu, dedicó el capítulo titulado "The danger within" de su libro Requiem for nature (1999) para examinar la presencia de los matsigenka de las comunidades nativas Tayakome y Yomibato como amenaza al PNM. Terborgh postula que debido a las necesidades que tienen los matsigenka de Tayakome y Yomibato de acceder a gasolina para operar sus botes con motores de $16 \mathrm{HP}^{8}$ y adquirir diferentes productos de origen manufacturero industrialartículos que no se pueden producir ni generar con la economía de subsistencia tradicional-entonces debían reubicarse en la parte externa, fuera del PNM ${ }^{9}$.

En su análisis comprensivo sobre el "arte de gobernar el Manu", Rodríguez (2018) ha examinado la mentalidad de gobierno (dialécticas control/amenaza y gobernantes/gobernados), el aparato institucional, la especialización (fronteras), las tecnologías y dispositivos de control y zonificación y las tensiones alrededor de la conservación y el desarrollo, que llevan, como resultante, a una situación de "ciudadanía graduada" dentro del PNM, que surge de la constatación de que el Estado "no trata a su territorio ni a sus ciudadanos de manera uniforme" (Rodríguez, 2018, p. 182). Con restricciones mayores para los sujetos que están más cerca de la naturaleza (pueblos indígenas). Lo que existe es un tejido ideológico para gobernar el parque basado en lo que la autora llama la "mantención de la tradicionalidad". "El arte de gobernar el Manu piensa, modela y fija la tradicionalidad-indigeneidad de maneras en que modos coloniales de entender la diferencia social continúan ejerciendo persistente influencia” (p. 186, énfasis original). En ese sentido, "conservar/gobernar el Manu implica la conformación de un orden político de excepción que produce espacios en términos de desarrollo y desigual y sujetos adecuados a éstos que pueden ser gobernados pero no pueden gobernar" (p. 189, énfasis original).

Dicho de otra forma, las políticas de conservación del PNM se hallan atrapadas en un tejido ideológico "proteccionista", cerrado a reinventar la gestión social y política del área protegida, especialmente para cumplir con los derechos de los pueblos indígenas, a pesar del cúmulo de mandatos y recomendaciones que,

\footnotetext{
${ }^{8} \mathrm{La}$ introducción de motores de 16HP a las comunidades matsigenka ocurrió a mediados de los ochenta. En 1983 el padre Ignacio Iraizos obtuvo una donación de un cooperante internacional de un motor de 16 HP (peque peque) para la comunidad de Tayakome en un intento de enganchar a dicha comunidad al aserradero que la misión dominica administraba entonces en Shintuya (Moore, 1984).

${ }^{9}$ En 1991, Panamericana Televisión (canal 5) divulgó la serie documental Manu, Reserva de Biósfera, que dirigió el periodista Alejandro Guerrero (https://www.youtube.com/watch?v=R45kQzHHmc4), mediante la cual la opinión de John Terborgh tuvo amplia cobertura. La actual versión en línea de esa serie documental es menos extensa y omite estas declaraciones.
} 
progresivamente, se han ido adoptando en espacios internacionales en los que se han abordado los asuntos relativos a áreas protegidas y pueblos indígenas ${ }^{10}$. Estos mandatos ${ }^{11}$ se han ido formulando para conciliar no solo la existencia de parques y la existencia de pueblos indígenas dentro de ellas, sino, también, para avanzar en otros asuntos cruciales como la gobernanza de ANP con la intervención directa de los propios pueblos indígenas. Con un sentido más amplio, merecen citarse también otros instrumentos internacionales: la Declaración de la ONU sobre los Derechos de los Pueblos Indígenas (2007) y la Declaración de la OEA sobre los Derechos de los Pueblos Indígenas (2016).

También existen recomendaciones de organismos de conservación para la observancia de derechos de pueblos indígenas en ANP. Por ejemplo, la Declaración del II Congreso Latinoamericano de Parques Nacionales y Otras

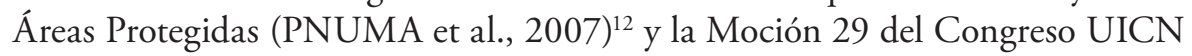
en Hawaii (2016) ${ }^{13}$.

\section{PlaneS ANTROPOlÓGICOS Y PROVISIONES SOBRE DERECHOS INDÍGENAS}

Los planes antropológicos han sido históricamente concebidos como una herramienta para la gestión de los asuntos indígenas dentro del PNM. Esto se refleja desde su primer Plan Maestro. Durante el proceso de constitución del PNM, entre 1970-1973, André-Marcel d'Ans desarrolló investigaciones sobre los indígenas del Manu en el marco de un convenio entre el Ministerio de Educación y la Universidad Nacional Mayor de San Marcos, instancia que entonces expresó "desde un inicio preocupación por la relación con la población indígena dentro del parque” (Helberg, 2001, p. 213). Ello inspiró las páginas del primer Plan Maestro del PNM dedicadas a algunos principios básicos para manejar la presencia de pueblos indígenas dentro del PNM, "como que la población indígena tiene libertad de movimiento y puede llevar a cabo sus actividades económicas tradicionales sin restricciones" (Helberg, 2001, p. 213).

Aunque estas provisiones de políticas antropológicas eran tributarias del enfoque paternalista y tradicionalista analizado por Rodríguez (2013, 2018), hubo de acaecer el contacto violento de los indígenas yora, que precipitó la

\footnotetext{
${ }^{10}$ Citamos, sintéticamente, algunos de estos mandatos: Convenio 169 de la OIT (aprobado en 1989, ratificado por el Perú en 1993 y vigente desde 1995), que requiere participacion de los pueblos indigenas en la administracion, uso y proteccion de recursos naturales y que declara como ilegal el reasentamiento forzado de pueblos nativos es ilegal; Convenio de Diversidad Biológica de 1992-1993, especialmente sus artículos 8j (conocimientos tradicionales relacionados con conservacion in situ) y 10c (uso sostenible de diversidad biológica); COP7 CDB Durban de 2004, Decisión VII/28 sobre áreas protegidas (participacion en el manejo de áreas protegidas, toma de decisiones, planificacion y gobierno); Directrices Akwe Kon (sobre evaluaciones de proyectos de desarrollo en tierras ocupadas o utilizadas tradicionalmente por comunidades indigenas); y COP10 CDB de 2010 (20 Metas de Aichi/Plan Estratégico para la Diversidad Biológica 2011-2020).

${ }^{11}$ En tanto son resultado de compromisos de Estados con caracter vinculante, que deben ser implementados.

${ }^{12}$ Respeto de los derechos de los pueblos indígenas en ANP superpuestas a territorios indígenas/ Concepto Territorios Indígenas de Conservación.

${ }^{13}$ En el cual se concertó el reconocimiento y respeto a los territorios y áreas conservadas por pueblos indígenas y comunidades locales que se solapan con áreas protegidas.
} 
presencia de un profesional antropólogo, para encarar este y otros asuntos indígenas del parque:

[...] recién en 1985, se reasume una conducción antropológica del Parque. El problema era que las comunidades andinas podían reclamar derechos sobre sus tierras y que era absolutamente inconveniente mantener una actitud tensa y conflictiva con ellos. Con los indígenas amazónicos, la relación con los guardaparques, excelente al inicio se había deteriorado. Además, los dos asentamientos matsigenka, Tayakome y Yomibato, tenían... muchas necesidades occidentales. Se habían conformado en torno a escuelas que habían modificado su patrón tradicional de asentamiento y ahora habían creado conflictos internos sobre territorios de caza que se sobreponen y un mayor impacto ambiental (Helberg, 2001, p. 213).

Dado que las "políticas iniciales eran proteccionistas y simplistas, había que reconstruir una relación positiva con el parque. Los indígenas amazónicos podían justificar racionalmente sus necesidades. Impedir la satisfacción de estas hubiera ido contra los derechos de la persona” (Helberg, 2001, 213). En 1985 se activó entonces un Departamento de Pueblos Nativos, con un antropólogo como responsable. Entre las funciones que se concibió para este estaban:

- Servir de nexo en la comunicación entre la población indígena del Parque con la administración del parque, autoridades y población que rodea al Parque, así como con la Sociedad Nacional.

- Diseñar las políticas antropológicas del Parque.

- Promover la participación de la población indígena en la administración del parque, asesorándolos en esta actividad.

- Buscar formas de comprensión y adecuación de sentido de un Parque Nacional a sus categorías de pensamiento.

- Representar los intereses de la población indígena no contactada ante la sociedad nacional en estrecha colaboración con la FENAMAD su legítima representante.

- Asumirá la activa defensa de los derechos indígenas (Helberg, 1987, pp. 3-4)

En 1985 este responsable promovió una reunión:

en que se da forma a un plan antropológico del Parque Nacional del Manu y en la que participan expertos y autoridades. El Parque necesitaba de un Departamento de Antropología capaz de servir de puente en el diálogo, con capacidad de negociar y de investigar. Esto, sin embargo, nunca le fue concedido, como también es cierto que las autoridades peruanas nunca vinieron a negociar con los mismos indígenas y así prevaleció una actitud autoritaria (Helberg, 2001, pp. 213-214).

En ese año el PNM, dependía, en mucho, para sus operaciones y pago del personal de la Corporación Departamental de Desarrollo de Madre de Dios (CORDEMAD). Esta instancia soportó muchas presiones hacia el trabajo del Departamento de Antropología y su personal. "De todo ello resultó que el joven Departamento de pueblos nativos se hizo políticamente inviable y fue 
desactivado. Se inició una nueva etapa de inactividad" (Helberg, 2001, p. 214). Esa fue la única vez en que el PNM contó con un plan antropológico, un equipo profesional y algunos recursos para funcionar. Con posterioridad se han producido otros planes antropológicos (INRENA, 2002b; Rummenhöller y Aguirre, 2007; Rummenhöller et al., 1997a) pero que nunca se aprobaron, mucho menos se buscó financiamiento para contratar personal especializado que permita implementarlos sistemáticamente.

Shepard et al. (2010) produjeron una línea de tiempo de los referidos planes antropológicos del PNM, que Rodríguez $(2018,101)$ cita, aunque en la perspectiva de Shepard et al. (2010) solo hubo dos nuevos planes (1997 y 2007). Rescato la lectura de las implicaciones que significaron estos otros instrumentos de gestión para los pueblos indígenas que Shepard y sus colegas señalan y que cito en la Tabla 3.

Sin embargo, es pertinente señalar la continuidad conceptual y de enfoque en la formulación de lineamientos de política para pueblos indígenas desde el segundo plan antropológico de 1997 hasta el último plan del 2007. Este último documento, reivindicó -como el que lo antecedió- los aportes conceptuales y de políticas antropológicas ${ }^{14}$, expresando "una mezcla de textos elaborados por los equipos encargados de los planes antropológicos del 1997, 2002 y 2007. No siempre fue posible marcar la contribución específica de cada equipo" (Rummenhöller y Aguirre, 2007, p. v) ${ }^{15}$.

Es pertinente destacar, además, que dentro de esta continuidad conceptual en los planes antropológicos de 1997, 2002 y 2007 se halla el análisis de mecanismos de compensación económica para pueblos indígenas que habitan en ANP por efectos de su conformación. El razonamiento seguido fue que, si el Estado, a nombre de la Nación, establece una ANP que causa directa o indirectamente perjuicios económicos a los pueblos indígenas, al no permitirles el desarrollo de actividades económicas comerciales por causa del carácter intangible del área que no lo permite, entonces se requiere compensar el daño económico causado. La fórmula, hasta ahora ensayada, para superar este conflicto, ha venido siendo la propuesta de "mecanismos que aseguren de manera equitativa la distribución de los costos y el acceso a los beneficios del área natural protegida, a fin de, con ello, generar la sustentabilidad necesaria para tornar viable el proceso de conservación perseguido al interior de un Parque Nacional, como el Manu”. La

\footnotetext{
${ }^{14}$ Las versiones de los planes antropológicos del 2002 y 2007 contienen en mucho mayor detalle lo que en este punto estamos tratando de modo acotado. Además, el plan antropológico del 2002 recogió las recomendaciones de la Defensoría del Pueblo sobre derechos indígenas dentro de las ANP, incluyendo sus derechos a reconocimiento legal, aprovechamiento de recursos naturales, divisiones profesionales especializadas para atender asuntos indígenas, entre otros, señalados por dicha fuente (Camino, 2002).

${ }^{15}$ Con la formulacion del Plan Antropológico 2002, el INRENA-ProManu promovió la preparación de un Plan de Contingencia para Pueblos Indígenas Aislados y en Contacto Inicial. Los anteriores planes (1987, 1997) incluían algunas pautas básicas para atender situaciones con PIACI. En el PNM, y mientras duró el Proyecto ProManu, el Plan de Contigencia PIACI 2002 se implementó con soporte financiero y logístico de dicho Proyecto.
} 
figura de "mecanismos de compensación" (Rummenhöller y Aguirre, 2007, p. 23) está contemplada en el Reglamento y el Plan Director de las ANP.

\section{Tabla 3.}

Planes antropológicos y provisiones para pueblos indígenas.

\begin{tabular}{|c|c|c|c|c|}
\hline Año & Autor/institución* & Nombre/ descripción & Implicaciones para comunidades & Estado \\
\hline 1968 & $\begin{array}{l}\text { Instituto Forest- } \\
\text { al La Molina }\end{array}$ & $\begin{array}{l}\text { Zonificación de } \\
\text { hábitats }\end{array}$ & $\begin{array}{l}\text { Recomienda varias zonas de uso } \\
\text { de recursos para las comuni- } \\
\text { dades nativas }\end{array}$ & $\begin{array}{l}\text { Nunca implemen- } \\
\text { tado }\end{array}$ \\
\hline 1970s & PNM & $\begin{array}{l}\text { Reglas informales } \\
\text { con respecto a } \\
\text { las poblaciones } \\
\text { nativas }\end{array}$ & $\begin{array}{l}\text { Poblaciones nativas son permi- } \\
\text { tidas de circular libremente al } \\
\text { interior y mantener actividades } \\
\text { de subsistencia "tradicionales" }\end{array}$ & $\begin{array}{l}\text { Seguidas hasta hoy } \\
\text { en gran medida }\end{array}$ \\
\hline 1986 & Ríos et al. & $\begin{array}{l}\text { Primer Plan } \\
\text { Maestro }\end{array}$ & $\begin{array}{l}\text { Muy poca información o planea- } \\
\text { miento antropológico: pobla- } \\
\text { ciones mantienen modo de vida } \\
\text { tradicional o dejan el parque }\end{array}$ & $\begin{array}{l}\text { Implementado en } \\
\text { líneas generales, } \\
\text { aunque lo referido } \\
\text { a las comunida- } \\
\text { des nunca les fue } \\
\text { comunicado }\end{array}$ \\
\hline 1989 & Helberg & $\begin{array}{l}\text { Primer Plan Antro- } \\
\text { pológico }\end{array}$ & $\begin{array}{l}\text { Lineamientos de política antro- } \\
\text { pológica y diálogo con comuni- } \\
\text { dades }\end{array}$ & $\begin{array}{l}\text { Nunca implemen- } \\
\text { tado }\end{array}$ \\
\hline $1997 a$ & $\begin{array}{l}\text { Rummenhöller, } \\
\text { Maldonado y } \\
\text { Castillo* }\end{array}$ & $\begin{array}{l}\text { Segundo Plan } \\
\text { Antropológico } \\
\text { (preliminar) }\end{array}$ & $\begin{array}{l}\text { Documento preliminar para coor- } \\
\text { dinar acciones, mejorar la calidad } \\
\text { de vida y promover la partici- } \\
\text { pación en la gestión del área }\end{array}$ & $\begin{array}{l}\text { Nunca implemen- } \\
\text { tado oficialmente, } \\
\text { pero algunas }\end{array}$ \\
\hline 1997b & $\begin{array}{l}\text { Rummenhöller, } \\
\text { Maldonado y } \\
\text { Castillo* }\end{array}$ & $\begin{array}{l}\text { Segundo Plan } \\
\text { Antropológico } \\
\text { (desarrollado)** }\end{array}$ & $\begin{array}{l}\text { Lineamientos de política antro- } \\
\text { pológica, normas** }\end{array}$ & $\begin{array}{l}\text { recomendaciones } \\
\text { adoptadas }\end{array}$ \\
\hline $2002 a$ & $\begin{array}{l}\text { INRENA y Pro- } \\
\text { Manu (Palma, } \\
\text { Ruiz Pereira y } \\
\text { Chávez)* }\end{array}$ & $\begin{array}{l}\text { Segundo Plan } \\
\text { Maestro }\end{array}$ & $\begin{array}{l}\text { Incorpora el desarrollo cultural } \\
\text { de los pueblos indígenas en los } \\
\text { objetivos del Parque Nacional }\end{array}$ & $\begin{array}{l}\text { Implementa- } \\
\text { do, aunque el } \\
\text { componente de } \\
\text { desarrollo fue el } \\
\text { mas descuidado }\end{array}$ \\
\hline $2002 b$ & $\begin{array}{l}\text { INRENA y Pro- } \\
\text { Manu (Pacheco, } \\
\text { García y Wahl)* }\end{array}$ & $\begin{array}{l}\text { Tercer plan antro- } \\
\text { pológico Revisión } \\
\text { y actualización } \\
\text { del Segundo Plan } \\
\text { Antropológico }\end{array}$ & $\begin{array}{l}\text { Garantizar los derechos territo- } \\
\text { riales indígenas en los territorios } \\
\text { del parque, mejorar el acceso a } \\
\text { educación y salud, participar en } \\
\text { la gestión del parque }\end{array}$ & $\begin{array}{l}\text { No implementado, } \\
\text { aunque sirve de } \\
\text { guía de la política } \\
\text { del PNM para } \\
\text { poblaciones }\end{array}$ \\
\hline 2003 & $\begin{array}{l}\text { Proyecto PRO- } \\
\text { MANU (Tello) }\end{array}$ & $\begin{array}{l}\text { Protocolo para } \\
\text { situaciones de con- } \\
\text { tacto con indígenas } \\
\text { aislados }\end{array}$ & $\begin{array}{l}\text { Referente para minimizar los } \\
\text { efectos negativos generados en } \\
\text { circunstancias de contacto con } \\
\text { indígenas aislados en el PNM y } \\
\text { RBM }\end{array}$ & $\begin{array}{l}\text { No implementado, } \\
\text { aunque sirve de } \\
\text { guía de la política } \\
\text { del PNM para } \\
\text { poblaciones }\end{array}$ \\
\hline 2007 & $\begin{array}{l}\text { Rummenhöller } \\
\text { y Aguirre }\end{array}$ & $\begin{array}{l}\text { Cuarto Plan } \\
\text { Antropológico. } \\
\text { Actualización de } \\
\text { lo elaborado por } \\
\text { INRENA y ProManu }\end{array}$ & $\begin{array}{l}\text { Recoge la mayoría de lo elabora- } \\
\text { do antes. Prioriza la actualización } \\
\text { del diagnóstico situacional de las } \\
\text { comunidades }\end{array}$ & No implementado \\
\hline 2007 & $\begin{array}{l}\text { Rummenhöller } \\
\text { y Huertas }\end{array}$ & $\begin{array}{l}\text { Plan de contingen- } \\
\text { cia en caso de con- } \\
\text { tacto con indígenas } \\
\text { en aislamiento } \\
\text { para el PNM }\end{array}$ & $\begin{array}{l}\text { Actualiza y amplía el documento } \\
\text { del Proyecto ProManu (2003) }\end{array}$ & $\begin{array}{l}\text { No implementado, } \\
\text { aunque sirve de } \\
\text { guía de la política } \\
\text { del PNM para } \\
\text { poblaciones }\end{array}$ \\
\hline 2013 & SERNANP & $\begin{array}{l}\text { Tercer Plan Mae- } \\
\text { stro 2013-2018 }\end{array}$ & $\begin{array}{l}\text { Plan antropológico es una activi- } \\
\text { dad opcional** }\end{array}$ & Nunca elaborado** \\
\hline
\end{tabular}

Fuente: Rodríguez (2018), Shepard et al. (2010). Elaboración propia, 2020. 
Cabe precisar que el Plan Maestro del PNM 2013-2018 contempló, como parte de su "estrategia de intervención" referida a "promover la inserción y articulación de los pueblos indígenas y la población como colaboradores estratégicos en la conservación del PNM" (Programa de Conservación de Recursos), la ejecución de la actividad "referencial": "promover la elaboración del Plan Antropológico del PNM en conjunto con el Viceministerio de Interculturalidad" (SERNANP, 2014, p. 67 $)^{16}$. Sucede que, desde julio del 2010, cuando se creó el Ministerio de Cultura, el SERNANP ha argumentado que los asuntos indígenas dentro del PNM son competencia del Ministerio de Cultura. El siguiente reporte del 2013, incluido en un diagnóstico de las capacidades institucionales del Estado peruano para afrontar la problemática de los pueblos indígenas aislados y/o en contacto inicial, en este caso, las relativas a las del SERNANP, dio cuenta de ello:

\begin{abstract}
Otros instrumentos de gestión corresponden a los Planes Antropológicos (para la presencia humana dentro de estas ANPs) y Planes de Contingencia (específicos para prevención relacionados a PIACI) para estas mismas ANPs y también para la Reserva Comunal Purús. Para su aprobación requieren de una normativa específica que ha sido trabajada con el VMI-MC y que actualmente está en proceso de revisión entre ambas instituciones (VMI-MC y SERNANP). Actualmente son documentos internos utilizados internamente por el personal de dichas ANPs con un enfoque preventivo y precautorio" (García, 2013, p. 36, énfasis original).
\end{abstract}

Sin embargo, no existe la normativa específica que el SERNANP anunció estar preparando con el Viceministerio de Interculturalidad. Por consiguiente, y a pesar de existir un Ministerio de Cultura, no existen lineamientos normativos para aprobar planes antropológicos ni planes de contingencia para PIACI (Soria, 2018) ${ }^{17}$.

En reemplazo de instrumentos y profesionales que se ocupen de los asuntos indígenas del PNM, existe un papel predominante de ONG e instituciones privadas en la gestión del PNM y el manejo de políticas con comunidades nativas. En realidad, el PNM depende de fondos de la cooperación técnica que siempre tienden a priorizar su propia agenda (Camino, 2010). Ejemplos históricos de ello son John Terborgh (Duke University en Cocha Cashu), San Diego Zoological Park y más recientemente Avisa Peru SZF.

\title{
AUTOGESTIÓN, DERECHOS Y PUNTOS DE VISTA INDÍGENAS
}

Ya hemos citado los pasajes de las políticas del Departamento de Pueblos Nativos de 1985-1986 para promover, sin mayor éxito, la autogestión indígena entre las comunidades del PNM, especialmente las comunidades Tayakome y

\footnotetext{
${ }^{16}$ Una nota de pie de página del Plan Maestro explica este atributo así: "Las actividades descritas se consideran referenciales, debido a que el contexto y entorno son cambiantes, no siendo de estricto cumplimiento".

${ }^{17}$ Además, de las omisiones funcionales del Ministerio de Cultura, existe una conducta errática por parte del SERNANP sobre qué normas de su legislación cumple y qué normas evita aplicar referidas a derechos de pueblos indígenas y de PIACI (Soria, 2018). Cabe seńalar que las Reservas Indígenas y las ANP pueden superponerse legalmente como es el caso de las reservas indígenas Mashco Piro (Parque Nacional Alto Purús) e Isconahua (Parque Nacional Sierra del Divisor), pero para el PNM el Ministerio de Cultura no ha formulado ninguna propuesta. Asimismo, al Ministerio de Cultura, como ente rector, le conciernen los problemas de las comunidades nativas dentro del PNM.
} 
Yomibato. Históricamente, algunos espacios de trabajo han hecho posible el desarrollo de capacidades y habilidades para la articulación de ideas, opiniones y planteamientos de los pueblos indígenas del PNM, en particular de las comunidades matsigenka articuladas a la sociedad, y del Estado. Digamos así, uno interno, de participación de las comunidades matsigenka del PNM en los Congresos de la FENAMAD, y otras actividades organizadas por ésta. Y otro externo, de participación de dichas comunidades en los espacios de debates en el Comité de Gestión de la Reserva de Biósfera del Manu y de Apoyo al PNM (CGRBM), junto con otras comunidades nativas colindantes con el PNM y otros actores sociales e institucionales, incluyendo la FENAMAD. Esto incluye la intervención de comunidades y organizaciones indígenas en las reuniones sobre el plan antropológico en sus sucesivos intentos de formulación.

La FENAMAD, entre 1985 y 1992 tuvo una participación relativamente permanente en las reuniones del CGRBM. En 1989, el VI Congreso de FENAMAD se llevó a cabo en la comunidad nativa de Shintuya, en el cual participaron delegados de Tayakome y Yomibato. Un grupo de indígenas nahua también llegó al Congreso. En esta reunión se trató un tema discutido también en las reuniones del CGRBM sobre el descontento de la comunidad de Tayakome por una investigación nutricional que implicaba mediciones de los alimentos ingestados. El Congreso aprobó un acuerdo exhortando al PNM a resolver esta situación ${ }^{18}$. Posteriormente, esta investigación fue trasladada de la Tayakome a Yomibato, por decisión de los propios investigadores (García, 2003).

En un taller realizado en Pakitza con la participación de investigadores y varias ONG y en el marco de la formulación del Plan Operativo 1992-1993 del $\mathrm{PNM}^{19}$, luego de una intensa discusión sobre la problemática de las comunidades matsigenka, se reconoció que el PNM se había establecido en forma inconsulta sobre el derecho territorial de los pueblos indígenas y por lo tanto era necesario reivindicar dicho derecho declarando al PNM como territorio indígena.

El plan antropológico de 1997 dio cuenta de las demandas de ambas comunidades que "solicitan un mayor control autogestionario sobre su territorio tradicional” (Rummenhöller et al., 1997, p. 47). Los planes del 2002 y 2007 citaron íntegramente la propuesta hecha en el taller de Pakitza de "declaración del PNM como territorio indígena” (Rummenhöller y Aguirre, 2007, p. 21). La persistencia de estos planteamientos señala la importancia del reconocimiento territorial indígena como una vía para resolver un tema neurálgico en la relación entre los pueblos indígenas y el PNM.

${ }^{18}$ El caso fortaleció las políticas de FENAMAD y de los Planes Antropológicos de 1997, 2002 y 2008 sobre investigadores e investigaciones en comunidades indígenas y derechos de propiedad intelectual.

${ }^{19}$ El primer Plan Maestro de 1985 estaba desactualizado. Se concibió formular un plan de menor jerarquía, pero bajo un enfoque participativo. Este plan contó con un amplio consenso social e institucional para su preparación. 
Como en los Congresos de FENAMAD siempre se ha discutido el tema territorial, emergió la propuesta de que deben titularse tierras en favor de las comunidades nativas del PNM. De allí que FENAMAD demandó dicha titulación en las dos primeras décadas del presente siglo. Esto que generó evidentemente reacciones de las esferas gubernamentales ligadas al PNM. Rodríguez y Feather (2014) han analizado, en efecto, la evolución de la perspectiva de los líderes matsigenka y de FENAMAD de una posición de demandar la exclusión de sus tierras del parque y el reconocimiento de títulos de tierras (como se entiende fuera de áreas protegidas, con muchos riesgos) a una posición más centrada en mecanismos legales para garantizar que se respeten sus derechos territoriales, además de medidas necesarias para abordar sus preocupaciones económicas, de salud y educación.

Ráez destaca que respecto de los derechos colectivos de los pueblos indígenas y sobre la gestión actual del PNM, si hay algún vacío que debe cubrirse es incorporar la voz de los mismos pueblos indígenas en torno de este asunto. FENAMAD remitió al SERNANP documentos elaborados en reuniones de trabajo del 2016 y 2017 por las comunidades matsigenka del PNM. Los contenidos de estos documentos bien pueden ser adoptados como ingredientes de la agenda indígena del PNM mas reciente. Las referencias, en estos documentos, a otros espacios de trabajo como el Mecanismo de Expertos sobre los Derechos de los Pueblos Indígenas (MEDPI) de las Naciones Unidas corresponde a la participación de un delegado matsigenka en una sesión de trabajo de ese año, como parte de la delegación de FENAMAD.

En el 2016, FENAMAD participó en el Congreso de la Unión Internacional de Conservación de la Naturaleza (UICN) realizado en Hawaii. Hasta ese año los miembros de la UICN eran estados y ONG, cuando se adoptó un acuerdo que permite, en adelante, que organizaciones de pueblos indígenas (OPI), adquieran la condición de miembros. Desde entonces, FENAMAD ha sido miembro de la UICN. También ha adquirido esa membresía el Ejecutor de Contrato de Administración de la Reserva Comunal Amarakaeri (ECA-RCA). FENAMAD es también punto focal para América del Sur de las OPI miembros de UICN. En ese proceso, FENAMAD se ha integrado igualmente al Consorcio TICCA, una red internacional de organizaciones de pueblos indígenas que conservan territorios indígenas que incluyen comunidades locales que no siendo pueblos indígenas conservan igualmente territorios indígenas.

En octubre del 2019 tuvo lugar el 3er Congreso de Parques Nacionales de Latinoamérica y El Caribe (Lima), evento que se reúne cada 10 años. Para este evento, FENAMAD, en su condición de Punto Focal para América del Sur de las OPI miembro de UICN, organizó un espacio de trabajo denominado Maloca Indígena, juntamente con otras organizaciones indígenas como AIDESEP y COICA y redes de trabajo como la instancia latinoamericana del Consorcio TICCA. En dicho foro ocurrieron presentaciones de representantes 
de organizaciones indígenas donde el tema derechos de los pueblos indígenas en ANP fue dominante.

En la delegación indígena de FENAMAD para el III CAPLAC participó un delegado matsigenka que señaló, entre otros aspectos, tres ideas centrales: declarar al PNM territorio indígena matsigenka y de otros pueblos remotos y aislados; establecer un Consejo Directivo del PNM que incluya, además de SERNANP, a los representantes de los pueblos indígenas presentes; y desarrollar un programa consensuado de fortalecimiento de los pueblos indígenas.

Las perspectivas y planteamientos de las comunidades matsigenka del PNM y de FENAMAD se enmarcan en el enfoque sobre gobernanza en ANP, entendida esta como el proceso de "tomar decisiones y garantizar las condiciones para su efectiva implementación" (Borrini-Feyerabend et al., 2014, p. 2) ${ }^{20}$.

\section{EsCENARIOS FUTUROS Y GOBERNANZA DEL PNM}

Dado que los pueblos indígenas del PNM están allí, no se los ha reubicado y han crecido ¿qué futuro se espera para el PNM? Shepard et al. (2013) han examinado esta situación, encontrando que los sistemas hortícolas y de caza de las comunidades matsigenka no son a futuro una amenaza para los ecosistemas que el PNM protege, concluyendo en una estrategia que llamaremos "ocupar la Amazonía" para reforzar la representación política de las comunidades y Federaciones indígenas, incluyendo a los Matsigenka del Manu como contrapeso a los intereses de recursos comerciales, haciéndolo de manera sostenible mejorando la salud y la educación de los indígenas y conservando sus estilos de vida. Es el deseo de las poblaciones indigenas mantener sus ecosistemas intactos. El ejercicio de los derechos legales de los indígenas protegerá a las reservas y parques nacionales frente a la explotación comercial a gran escala, la construcción de carreteras y el recorte de su territorio (2013, pp. 342-343, énfasis original).

Esta perspectiva parece admitir que se propone, de alguna forma, el reconocimiento legal de derechos territoriales indígenas dentro del parque. Sobre esto, Rodríguez y Feather plantean lo siguiente: "Los habitantes indígenas de Manu son titulares de derechos y no simplemente otra parte interesada cuyos intereses pueden intercambiarse con otras prioridades. El Parque Nacional del Manu es principalmente su hogar, más que una atracción turística o un punto caliente de biodiversidad" (2014, p. 483).

Actualmente el tema de la gobernanza del PNM está planteado, en alguna forma, conforme han concluido varias investigaciones sobre la urgencia de una reingeniería institucional. Así, "el aparato institucional que sostiene el gobierno del Manu necesita ser reformado no para incluir aspectos o áreas sociales, sino en función del reconocimiento de que la conservación es antes que nada un acto

${ }^{20}$ Ver también el Informe de Política del Consorcio TICCA 5 (Farvar et al., 2018). 
de regulación de procesos socioespaciales antes que naturales" (Rodríguez, 2018, p. 193). Se necesita:

quebrar la dicotomía entre sujetos gobernados y gobernantes si se va a tomar en cuenta seriamente consideraciones de justicia social. Esto implica ir más allá de la apertura de espacios de participación de los indígenas en los mecanismos de toma de decisiones, sino fundamentalmente la incorporación de sus modos de imaginar la naturaleza y la indigeneidad-tradicionalidad, así como incorporar las lógicas particulares de sus instituciones de manejo de recursos comunes, sus economías, y sus historias. Es necesario, en pocas palabras, restaurar presencias (Rodríguez, 2018, p. 193, énfasis original).

En la historia del PNM, esta discusión sobre la necesidad de refundarlo sobre nuevas bases ya ocurrió, al menos una vez, en los debates públicos a propósito del Plan Operativo 1992-1993. Cuando se propugnó un consejo directivo que conduzca la institución, con participación de representantes sociales e institucionales, incluyendo lo que ahora vendría a ser el SERNANP. En ese entonces, ese consejo directivo sería quien designa al jefe del área protegida (Arce y García, 1995).

En las recomendaciones de Ráez sobre el tema, este propone "una instancia de toma de decisiones sobre las condiciones y el régimen de vida entre los habitantes del Manu, donde participen protagónicamente los propios matsigenka nativos del área protegida. Naturalmente, el SERNANP y el Ministerio de Cultura formarían parte esencial de esta instancia” (Ráez, 2018, p. 49), a la cual se debe agregar, "una instancia ejecutiva también con participación directiva de los nativos del Manu, que lleve a cabo y supervise las decisiones alcanzadas" (p. 50). Este autor precisa que este planteamiento es compatible con la legislación peruana, en referencia al concepto de cogestión de áreas protegidas ya adoptado por el Estado. La cogestión ha avanzado especialmente en Reservas Comunales, como es el caso de la Reserva Comunal Amarakaeri, vecina del PNM. Sin embargo, las discusiones sobre el diseño institucional futuro del PNM no parecen ligarse al reconocimiento de los derechos indígenas, especialmente, los territoriales, que permitan una base distinta para refundar el parque, si ello quisiera volver a intentarse.

\section{A MOdo DE CONCLUSIón}

Existe un mosaico de situaciones socioculturales de los pueblos indígenas, desde comunidades articuladas a la sociedad occidental hasta pueblos indígenas aislados, cuya adecuada caracterización es relevante para comprender y orientar el trabajo con o sobre ellos. Los planes antropológicos han provisto políticas, pautas, diagnósticos, planes de trabajo y otras provisiones para apoyar a los pueblos indígenas del PNM respetando sus derechos y su organicidad mediante FENAMAD. Sin embargo, salvo la experiencia de 1989, el resto de planes antropológicos nunca fueron oficialmente aprobados, menos financiados y, por tanto, no ejecutados. Desde el 2010 en que existe el Ministerio de Cultura, en 
vez de haberse fortalecido esta dimensión de la gestión pública a favor de los pueblos indígenas del PNM, incluyendo los pueblos en aislamiento y contacto inicial, planes antropológicos y planes de contingencia (para pueblos indígenas aislados y en contacto inicial) han caído en un vacío normativo.

Existen contradicciones en la gestión del PNM de cara a los pueblos que la habitan. Los factores detrás de esas contradicciones que mantienen atrapado al PNM para avanzar en refundar su gestión de cara a sus pueblos indígenas se hallan en su matriz conceptual instalada en la dicotomía naturaleza/sociedad que preconiza el "proteccionismo" de diversidad biológica y teniendo a sus propios pueblos indígenas como amenaza. Aunque nuevos análisis están documentando que dicha amenaza no existe, al menos en lo que concierne a impactos futuros por crecimiento de población para las actividades de la economía indígena, un campo creciente de problemas se crea alrededor de los arreglos económicos de las comunidades matsigenka en su articulación con la economía de mercado, en el contexto de proyectos de carreteras impulsados por grupos de poder local en las puertas del PNM.

Mientras el gobierno del PNM, desde la perspectiva estatal, intenta mantener un statu quo, prolongando, en el tiempo, el encaramiento de las contradicciones subyacentes, las reclamaciones de las comunidades matsigenka, articuladas políticamente por sus organizaciones representativas, incluyendo FENAMAD, están procurando un abordaje de la situación, comprendiendo que si bien es bienvenida la conservación de diversidad biológica, este fin debe perseguirse respetando y observando los derechos de los pueblos indígenas, incluyendo sus derechos territoriales. En ese camino, las comunidades matsigenka del PNM y FENAMAD convergen actualmente con los enfoques de gobernanza y conservación inclusiva, que asumen, resultante de la articulación de FENAMAD como miembro de la UICN y del Consorcio TICCA.

En Bolivia, con texto constitucional similar al peruano en lo que refiere a la propiedad del territorio con cobertura forestal y de ANP, se han declarado varios Parques Nacionales y Territorios Indígenas, destacándose el Parque Nacional Madidi en la frontera con el Perú, que pueden tomarse en cuenta como referente, para articular territorios indígenas con ANP.

Sin embargo, existen riesgos de que los ensayos que podrían emprenderse alrededor de la cogestión indígena del PNM se realicen "secuestrando" y cooptando a representantes indígenas que luego son absorbidos e integrados al sistema administrativo del SERNANP. Siguiendo a Giuseppe Tomasi di Lampedusa, en su novela $E l$ Gatopardo, donde dice: "Que todo cambie para que todo siga igual", podemos decir que existen experiencias como el uso discrecional de la autonomía de las comunidades nativas (casos de negociaciones con empresas 
petroleras) donde terceros han excluido a sus organizaciones representativas, de las negociaciones y procesos, con el consiguiente divisionismo interno y nuevos conflictos.

\section{Agradecimientos}

Este artículo se beneficia de la participación del autor en el evento académico SEPIA XVII (Cajamarca, agosto de 2017). Comentarios que permitieron mejorar la versión inicial de este artículo fueron realizados por Julio Cusurichi, María Ponce, Thomas Moore, Héctor Sueyo, Heinrich Helberg, Klaus Rummenhöller, Miguel Alexiades y Daniel Rodríguez. Una versión mejorada de este artículo se benefició de las observaciones críticas de Danny Pinedo, profesor de la Escuela de Antropología de la Universidad Nacional Mayor de San Marcos (UNMSM). Agradezco a todos los comentarios. El mapa de este artículo fue preparado por FENAMAD (2016). Igualmente, mis agradecimientos a FENAMAD por esta colaboración y por su permiso para citar información procedente de reuniones de trabajo con comunidades matsigenka. La responsabilidad de su contenido es mía.

\section{REFERENCIAS}

Arce, Rodrigo y García, Alfredo (1995). Impacto social y ambiental de la Zona Reservada Tambopata-Candamo (ZRTC) en el desarrollo sostenible de las comunidades locales en el suroriente peruano (documento de trabajo). Puerto Maldonado: Centro Eori, FCFMA-UNSAAC.

Borrini-Feyerabend, G.; Bueno, P.; Hay-Edie, T.; Lang, B.; Rastogui, A. y Sandwith, T. (2014). Cartilla sobre gobernanza para áreas protegidas y conservadas. Linea temática sobre gobernanza del Congreso Mundial de Parques 2014 de la UICN. Gland (Suiza): UICN.

Camino, Alejandro. (2000). Población indigena y áreas protegidas: patrimonios resguardados, intereses en conflicto. Lima: Defensoría del Pueblo.

Consejo Transitorio de Administración Regional Madre de Dios [CTAR] \& Instituto de Investigaciones de la Amazonía Peruana [IIAP] (1999). Mapa de poblaciones indigenas (Proyecto Zonificación Ecológico-económica de la Región Madre de Dios). Manuscrito inédito.

Dávila, G. y Montoya, V. (1999). Prevención social y estimación de riesgos en la cuenca alta del río Las Piedras, Madre de Dios. Lima: Mobil Exploration and Producing Peru Inc. MEPPI. Manuscrito inédito.

Farvar, M. T.; Borrini-Feyerabend, Grazia; Campese, Jessica; Jaeger, Tilman; Jonas, Holly y Stevens, Stan (2018). Conservación inclusiva ¿¿de quién? (informe No. 5). Teherán: Consorcio TICCA y CENESTA.

Federación Nativa del Río Madre de Dios y Afluentes [FENAMAD] (2002). Algunas evidencias de la presencia de pueblos indigenas en situación de aislamiento en los rios Los Amigos, Las Piedras, Tahuamanu, Yaco y afluentes en Madre de Dios (de 1980 al 2002). Manuscrito inédito. 
García, Alfredo (1998). Análisis de la ocupación y transformación del espacio generada por diferentes actividades productivas-extractivas en la región de Madre de Dios. Informe final. Puerto Maldonado: Instituto de Investigaciones de la Amazonía Peruana [IIAP].

García, Alfredo (2003). FENAMAD 20 años después: Apuntes sobre el movimiento indígena amazónico en Madre de Dios. En Beatriz Huertas Castillo y Alfredo García Altamirano. (Eds.), Los pueblos indígenas de Madre de Dios: Historia, etnografía y coyuntura (pp. 274-309). Lima: Grupo Internacional de Trabajo sobre Asuntos Indígenas [IWGIA].

García, Alfredo (Ed.). (2013). Diagnóstico institucional Perú para la protección de los pueblos indígenas en situación de aislamiento y contacto inicial. Documento Técnico. Lima: Ministerio de Relaciones Exteriores \& Ministerio de Cultura, Viceministerio de Interculturalidad.

Gray, Andrew (1992). Entre la integridad cultural y la asimilación: Conservación de la biodiversidad y su impacto sobre los pueblos indigenas. Copenhague: Grupo Internacional de Trabajo sobre Asuntos Indígenas [IWGIA].

Helberg, Heinrich (1987). Departamento de antropología del Parque Nacional del Manu (documento de trabajo). Cusco: Jefatura del Parque Nacional del Manu.

Helberg, Heinrich (2001). Política con poblaciones en el Parque Nacional del Manu. En Lily Rodríguez. (Ed), El Manu y otras experiencias de investigación $y$ manejo de Bosques (pp. 213-214). Cusco: ProManu, UNESCO.

Huertas Castillo, Beatriz (2002). Los pueblos indigenas en aislamiento: Su lucha por la sobrevivencia y la libertad. Lima: Grupo Internacional de Trabajo sobre Asuntos Indígenas [IWGIA].

Huertas Castillo, Beatriz (2015). Corredor territorial de los pueblos indigenas en aislamiento y contacto inicial pano, arawaky otros. Lima: Asociación Interétnica de Desarrollo de la Selva Peruana [AIDESEP], Organización Regional de AIDESEP-Ucayali [ORAU], Coordinadora de Pueblos Indígenas de Atalaya [CORPIAA], Consejo Machiguenga del Rio Urubamba [COMARU], Federación Nativa del Río Madre de Dios y Afluentes [FENAMAD], Organización Regional de los Pueblos Indígenas del Oriente [ORPIO].

Instituto Nacional de Recursos Naturales [INRENA] \& ProManu. (2002). Plan antropológico del Parque Nacional del Manu (documento de trabajo). Cusco.

Ministerio de Agricultura [MINAGRI] (2000). Directorio de comunidades nativas del Perú 1999. Proyecto Especial de Titulación de Tierras y Catastro Rural. Lima: Proyecto Especial de Titulación de Tierras (PETT), Proyecto Asesoría en Planeación Agraria (PROAPA), Gesellschaft für Technische Zusammenarbeit (GTZ).

Moore, Thomas (1984a). Peru. Manu National Park and indigenous peoples endangered. IWGIA Newsletter, (37), 126-135.

Programa de las Naciones Unidas para el Medio Ambiente [PNUMA], Organización de las Naciones Unidas para la Agricultura y la Alimentación [FAO], Unión 
Mundial para la Naturaleza [UICN], Comisión Mundial de Áreas Protegidas [CMAP], Redparques y Administración de Parques Nacionales de Argentina [APN] (2007). Declaración de Bariloche. II Congreso Latinoamericano de Parques Nacionales y otras áreas protegidas. San Carlos de Bariloche, Argentina.

Raez-Luna, Ernesto (2018). Pueblos indígenas y conservacion de la naturaleza: ¿Debates teóricos o urgencias vitales? El caso del Parque Nacional del Manu. En Ernesto Raez-Luna. (Ed.), Derechos ancestrales y conservación de la naturaleza en debate: El caso de los pueblos indígenas del Parque Nacional del Manu (pp. 13-57). Lima: Seminario Permanente de Investigación Agraria [SEPIA].

Reynoso Vizcaino, Patricia y Helberg Chávez, Heinrich (1986). Primer rstudio Etnográfico del grupo étnico yura o nahua. Manuscrito inédito.

Ríos, Manuel; Vásquez, Pedro; Ponce, Carlos; Tovar, Antonio y Dourojeanni, Marc (1985). Plan maestro: Parque Nacional del Manu. Lima: Universidad Nacional Agraria La Molina.

Rodríguez Castañeda, Sandra (2013). La naturalización de la naturaleza: Discursos y politicas de conservación en el Parque Nacional del Manu. Seminario Bienal SEPIA XV. Chachapoyas, Perú.

Rodríguez Castañeda, Sandra (2018). Conservar la naturalezalgobernar la población. Imaginario, espacio y politicas en el Parque Nacional del Manu [Tesis de licenciatura]. Pontificia Universidad Católica del Perú, Lima.

Rodríguez, Daniel y Feather, Conrad (2014). A refuge for people and biodiversity: The case of Manu National Park, South-East Peru. En Stefan Risko y Helen Tugendhat. (Eds.). World heritage sites and indigenous people's rights (pp. 459-526). Copenhagen: Grupo Internacional de Trabajo sobre Asuntos Indígenas [IWGIA]:

Rummenhöller, Klaus (1995). Territorios indígenas y áreas naturales protegidas. En Organización Nacional Indígena de Colombia [ONIC], Centro de Cooperación al Indígena [CECOIN] y GhK ELNI. Tierra profanada: Grandes proyectos en territorios indigenas de Colombia (pp. 65-90). Bogotá: Disloque Editores.

Rummenhöller, Klaus y Aguirre, Indira (2007). Plan antropológico para el Parque Nacional del Manu elaborado con alcance a la Reserva de Biósfera del Manu (2008-2012), tomo 1 (Documento sobre lineamientos políticos, conceptos, componentes estratégicos y operativos). Lima.

Rummenhöller, Klaus; Maldonado, Waldo y Castillo, Ada (1997a). Plan antropológico y de promoción social del Parque Nacional del Manu (documento de trabajo). Cusco.

Rummenhöller, Klaus; Maldonado, Waldo y Castillo, Ada (1997b). Bases para la planificación y desarrollo de las comunidades andinas y amazónicas en la Reserva de Biosfera del Manu. Lima: Instituto Nacional de Recursos Naturales [INRENA] y TCA-SURAPA. 
Servicio Nacional de Áreas Naturales Protegidas [SERNANP] (2014). Plan maestro Parque Nacional del Manu 2013-2018 (documento de trabajo). Lima.

Shepard, Glenn (1996a). Informe 1: Los grupos indígenas aislados del río Piedras. Informe presentado a Mobil Exploration and Producing Perú Inc.-Sucursal peruana (MEPPI). Manuscrito inédito.

Shepard, Glenn (1996b). Informe 2: Visita al campamento base, rio de las Piedras, 3-7 octubre. Informe presentado a Mobil Exploration and Producing Perú Inc.Sucursal peruana (MEPPI). Manuscrito inédito.

Shepard, Glenn H, Jr.; Rummenhöller, Klaus; Ohl-Schacherer, Julia; Yu, Douglas W. (2010). Trouble in paradise: indigenous populations, anthropological policies, and biological diversity conservation in Manu National Park, Peru. Journal of Sustainable Forestry, 29(2-4), 252-301.

Shepard, Glenn; Yu, Douglas; Ohl-Schareder, Julia y Levi, Taal (2013). Resolviendo el conflicto "parque-personas" en el Manu, con la estrategia "ocupar la amazonía”. En Jessica Groenendijk y Antonio Tovar. (Eds.). Reporte Manu 2013: Pasión por la investigación en la Amazonía Peruana (pp. 342-369). Lima: San Diego Zoo Global Peru.

Soria Dall'Orso, Carlos (2018). Realidad y desafios de la legislación de protección de pueblos indígenas en aislamiento voluntario y contacto inicial que viven dentro de áreas naturales protegidas. En Ernesto Raez-Luna. (Ed.), Derechos ancestrales y conservación de la naturaleza en debate: El caso de los pueblos indigenas del Parque Nacional del Manu (pp. 61-107). Lima: Seminario Permanente de Investigación Agraria [SEPIA].

Terborg, John (1999). Requiem for nature. Washington, D.C.: Island Press, Shearwater Books.

Wahl, Lissie (1988). El Manu, los nahua y Sepahua frente a la madera: Ideología y producción. Perú Indigena, (29), 145-170. 